\title{
The Utility of Immunohistochemistry for Mismatch Repair Proteins on Colorectal Polyps in the Familial Cancer Clinic
}

$\operatorname{Dr}_{\text {Eryn Dow }}{ }^{1 \mathrm{a}}, \mathrm{A} /$ Prof Daniel D. Buchanan ${ }^{1,2,3,4}$ \& Prof Ingrid M. Winship ${ }^{1,5}$

${ }^{1}$ Genetic Medicine and Family Cancer Clinic, Royal Melbourne Hospital, Parkville, Australia

${ }^{2}$ Colorectal Oncogenomics Group, Genetic Epidemiology Laboratory, Department of Pathology, The University of Melbourne, Parkville, Victoria, Australia

${ }^{3}$ Centre for Epidemiology and Biostatistics, Melbourne School of Population and Global Health, The University of Melbourne, Parkville, Victoria, Australia

${ }^{4}$ University of Melbourne Centre for Cancer Research, Victorian Comprehensive Cancer Centre, Parkville, Victoria, Australia

5 Department of Medicine, The University of Melbourne, Parkville, Victoria, Australia

\section{Corresponding Author}

Eryn Dow

Genomic Medicine and Family Cancer Clinic

Level 2 Centre, The Royal Melbourne Hospital

300 Grattan Street, Parkville, Australia 3050

egdow@hotmail.com

$+61404158854$

\section{Acknowledgements}

No funding was received to support this study. The authors declare that they have no competing interests and acknowledge no other contributors to this work. All authors read, contributed to the writing of, and approved the final manuscript; Eryn Dow collected and analysed the data.

\section{Word Count}

Abstract - 250, Main Text - 2763

\footnotetext{
${ }^{a}$ Current Address: Clinical Genetics Service, Austin Hospital, 145 Studley Road, PO Box 5555 Heidelberg, Victoria, Australia 3084
}

This is the author manuscript accepted for publication and has undergone full peer review but has not been through the copyediting, typesetting, pagination and proofreading process, which may lead to differences between this version and the Version of Record. Please cite this article as doi: 10.1111/imj.13953 
Keywords: Polyps, DNA Mismatch Repair, Immunohistochemistry, Colorectal Neoplasm, Hereditary Nonpolyposis

This article is protected by copyright. All rights reserved. 


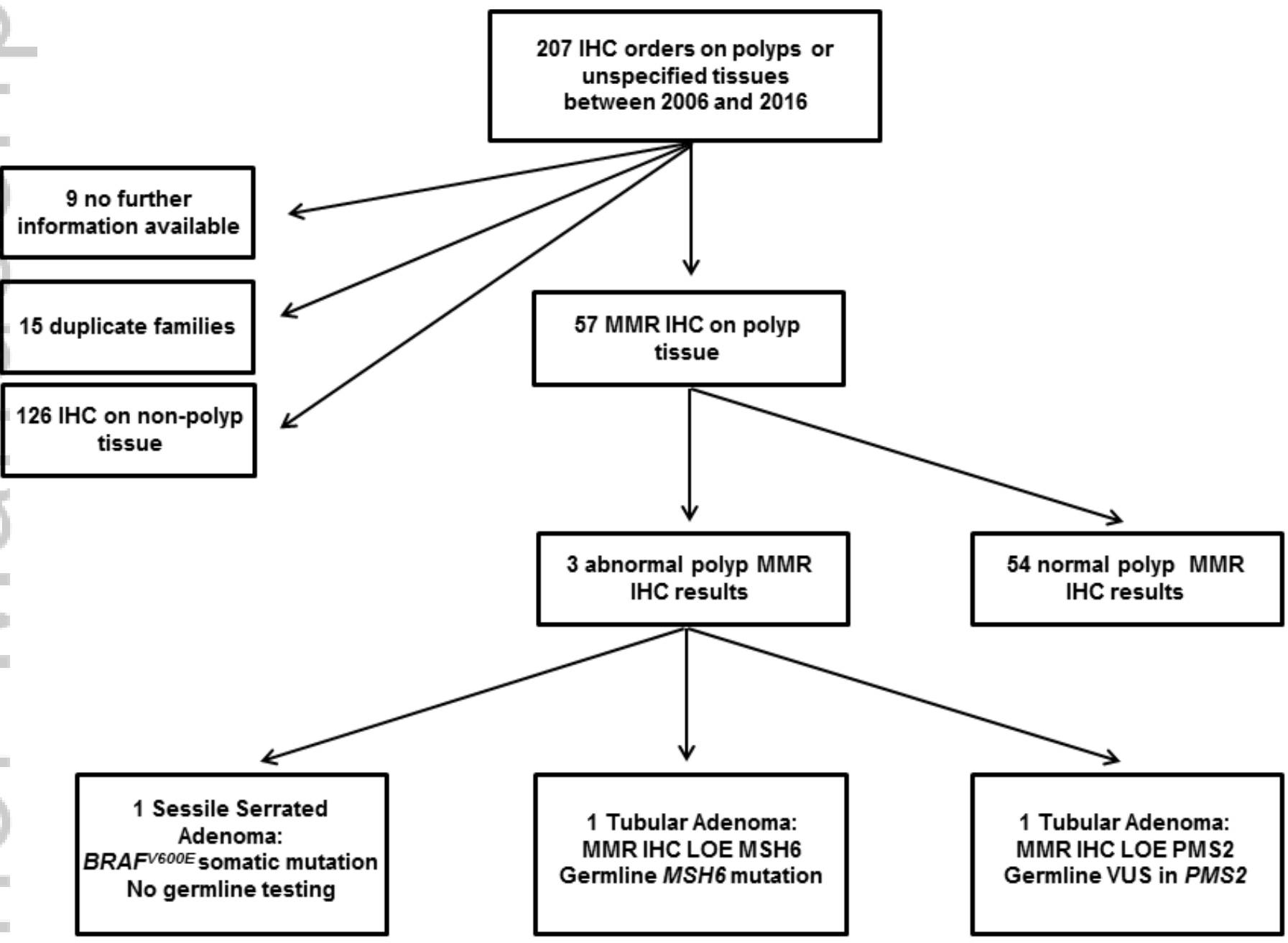

Figure 1.tif

This article is protected by copyright. All rights reserved. 


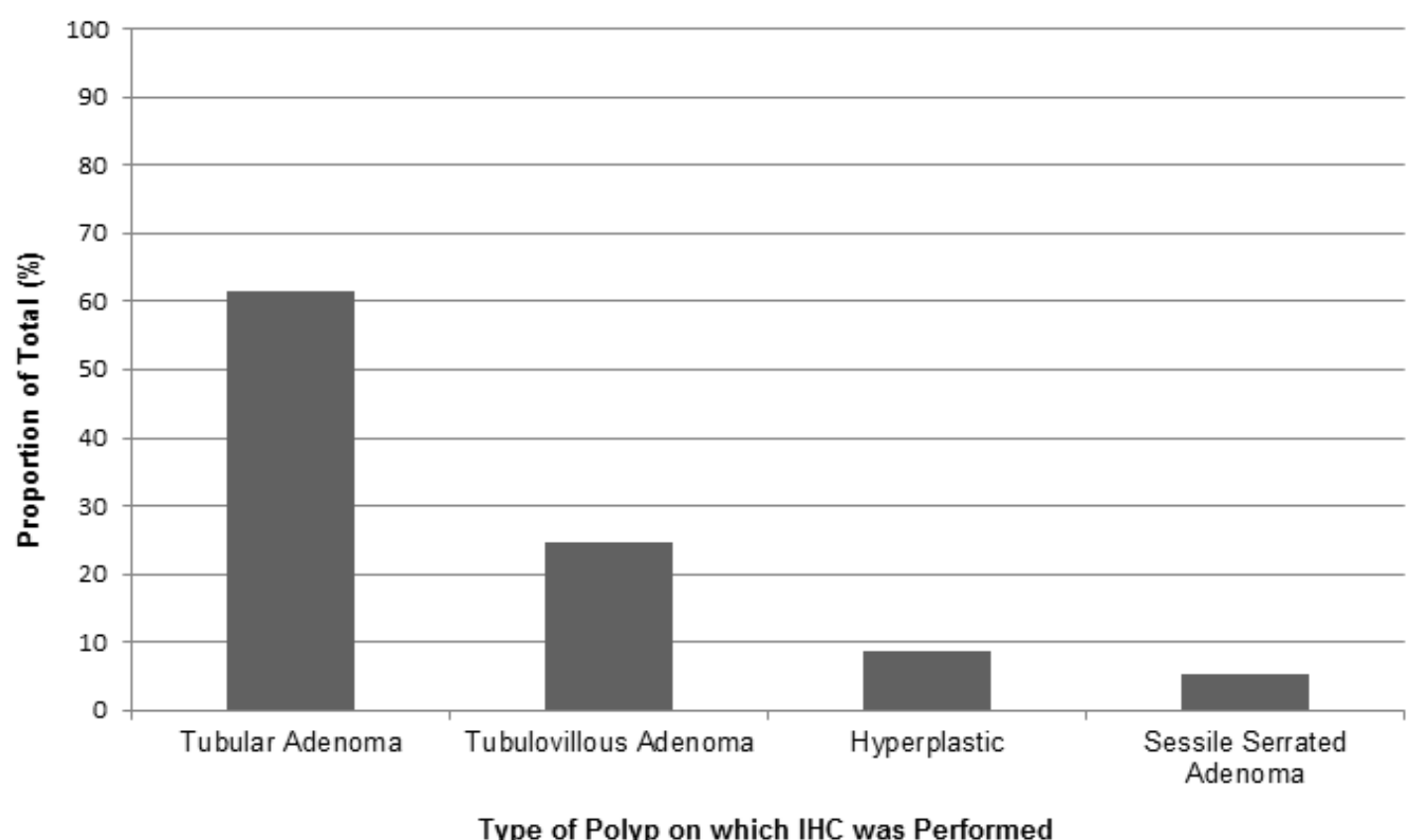

Figure 2.tif

This article is protected by copyright. All rights reserved. 


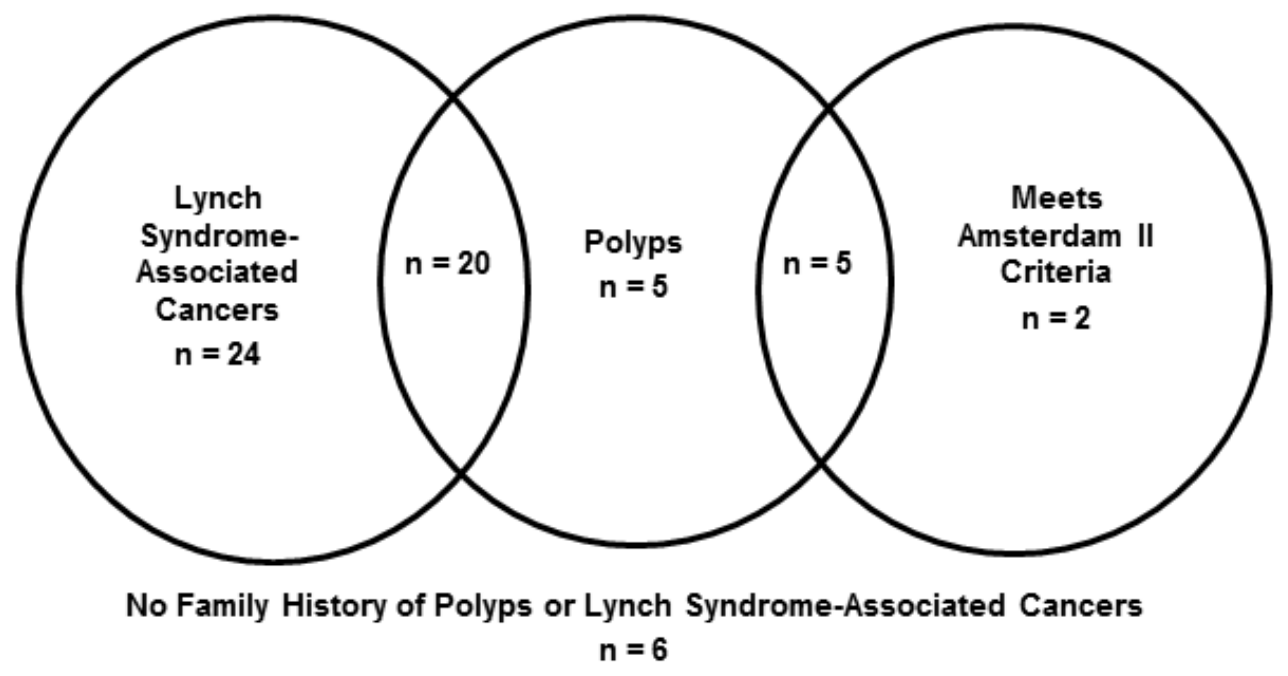

Figure 3.tif

This article is protected by copyright. All rights reserved. 


\section{$\underline{\text { The Utility of Immunohistochemistry for Mismatch Repair Proteins on Colorectal }}$}

\section{Polyps in the Familial Cancer Clinic}

\section{Introduction}

Lynch Syndrome (LS), caused by autosomal dominant inheritance of a germline mutation in one of the four DNA mismatch repair (MMR) genes (MLH1, MSH2, MSH6, PMS2), or within the EPCAM gene, results in an increased incidence of malignancy, predominantly colorectal and endometrial cancers. The MMR genes normally function to correct mutations resultant from DNA replication, particularly in repetitive sequences like microsatellites ${ }^{1,2}$. Immunohistochemistry (IHC) for loss of expression (LOE) of one or more of the MMR proteins can be performed on malignant tissue as a screening test for LS; subsequent germline genetic testing, guided by the pattern of MMR LOE, confirms LS. Alternatively, microsatellite instability (MSI) testing of malignant tissue can screen for LS as loss of MMR function results in increased mutational burden within the microsatellites. However, in contrast to MMR IHC, MSI testing does not indicate which MMR gene may be causative.

Colorectal cancers in LS are believed to arise via the adenoma-carcinoma pathway where a polyp precedes invasive malignancy, but at a younger ages and/or accelerated rates compared to sporadic cancers ${ }^{3}$. This raises the possibility of performing MMR IHC on premalignant polyps as a screening tool for LS, particularly as an early detection strategy in young patients. Identifying individuals with LS can be clinically challenging due to the lack of phenotypic signs preceding cancer and variable penetrance in families ${ }^{4}$. However, identification of LS families is vital for cancer risk management through surveillance, and/or risk reducing strategies $^{5}$.

This article is protected by copyright. All rights reserved. 
Previous studies of polyp MMR IHC have yielded mixed results. Adenomas from LS patients have rates of abnormal MMR IHC from $50 \%$ to $80 \%{ }^{4,6,7}$, and rates of MSI from $57 \%$ to $93 \%{ }^{8}$. Adenomas which are larger in size $(>8 \mathrm{~mm})^{7}$, contained high-grade dysplasia, and/or had a villous component were more likely to show $\mathrm{LOE}^{4}$. Similarly, a significant association between high grade dysplasia and high levels of MSI has been demonstrated ${ }^{8}$. The specific LS gene may also impact polyp MMR IHC, with MLH1 LOE most and MSH6 LOE least commonly detected (over $90 \%$ versus $36 \%$, respectively) ${ }^{4}$. However, these studies were all carried out in LS patients and provide little insight on the use of polyp MMR IHC in screening for LS. In an unselected Finnish population, the frequency of MSI in adenomas was only $1.6 \%{ }^{9}$. A recent study demonstrated MMR IHC performed on adenomas from young patients (age $<50$ years) not known to have LS, nor having a suggestive family history, was abnormal in only $0.4 \%$ of cases ${ }^{5}$. While adenoma MSI testing in a young $(<40$ years old) Portuguese cohort found that MSI correlated with the identification of a germline LS mutation in $85.7 \%$ cases, though all cases had Amsterdam Criteria-positive family histories ${ }^{10}$. This suggests the rate of abnormal MMR IHC or MSI in sporadic adenomatous polyps is low $(<2 \%)^{9,11,12}$, and that screening of polyps may be effective in identifying new cases of $\operatorname{LS}^{11,12}$.

With the current healthcare focus on effective use of funding, the clinical value and utility of any test or treatment must be carefully considered. This is embodied by the Royal Australasian College of Physician's Evolve Program ${ }^{13}$, based upon the American Board of Internal Medicine's Choosing Wisely Initiative ${ }^{14}$. These programs encourage the identification and reduction or avoidance of low value medical practices, aiming to provide the highest quality and value care ${ }^{13}$. In this setting, evaluating the role of MMR IHC on polyps, with its associated cost and unclear clinical benefit is relevant. This retrospective 
audit was conducted to determine the clinical utility of MMR IHC performed on nonmalignant polyps in patients attending a Familial Cancer Centre (FCC).

\section{Methods}

A retrospective audit identifying all patients attending the FCC at the Royal Melbourne Hospital between 2006 and 2016 for whom MMR IHC was ordered on pre-malignant polyps was conducted. Their paper and electronic medical records were reviewed, collecting information on family and personal malignant history, MMR IHC results from personal or familial tissue and germline genetic testing results, where available. This study was approved by the Melbourne Health Human Research Ethics Committee.

A database query of all IHC orders on either polyp or unspecified tissue over the ten year period identified 206 patients. After review of all records, 57 patients met the inclusion criteria of having had IHC ordered on non-malignant colorectal polyp tissue; testing was requested by varying FCC-associated clinicians. Patients were excluded due to: MMR IHC on malignant polyps or other malignant tissue (colorectal, endometrial, or ovarian cancers), IHC for non-MMR proteins (SDHB for example), or familial duplicates (15 patients). An additional 9 patients were excluded as their records were unavailable for review (Figure 1). Patients diagnosed with LS were not excluded; however, as these patients preferentially proceeded directly to germline genetic testing, no requests for polyp MMR IHC in patients with LS were identified.

MMR IHC was performed by the Pathology Department at the Royal Melbourne Hospital using accredited protocols and antibodies. Genetic testing was carried out by the accredited Victorian Clinical Genetics Service or Peter MacCallum Cancer Centre Molecular 
Laboratory, including MMR gene panel testing (MLH1, MSH2, MSH6, PMS2), or MUTYH and/or $A P C$ single gene testing, as determined by the treating clinician. As genetic testing evolved, multi-gene panels (MUTYH and $A P C$, or a Colorectal Cancer and Polyposis 18 gene panel) were utilised. Whole exome sequencing was performed in one patient as part of a research program through the Melbourne Genomic Health Alliance (http://www.melbournegenomics.org.au).

\section{Results}

We identified 57 patients who underwent polyp MMR IHC; the average age at the time of testing was 53 years (range 18-81). The median number of polyps per patient was three, with a range of one to $>100$. Thirty-three patients had tubular adenomatous polyps (TA) or tubulovillous adenomatous polyps (TVA), 20 patients had mixed TA/TVA and Hyperplastic polyps (HP), two patients had HP polyps only, one patient had only Sessile Serrated Adenomatous polyps (SSA), and one patient had mixed SSA and HP. MMR IHC was performed on a TA or TVA in $61.4 \%(n=35)$ and $24.6 \%(n=14)$ of cases, respectively; five (8.8\%) and three (5.3\%) patients, respectively, had MMR IHC on a HP or SSA (Figure 2). Fifty-six of the 57 patients had polyp MMR IHC on only one polyp; one patient had MMR IHC on two polyps (1 TA, $1 \mathrm{HP})$.

A personal history of synchronous or metachronous LS-associated cancers was identified in seven patients, six colorectal cancers and one endometrial cancer. In five of the six patients with colorectal cancers, MMR IHC on tumour tissue was concordant with polyp testing; in one case tumour tissue was unavailable due to the interval since diagnosis ( 37 years). Tumour tissue was also unavailable for the patient with metachronous endometrial cancer. Two patients had a personal history of breast cancer and one patient had a personal history of 
prostate cancer, MMR IHC performed on the prostate cancer was normal and concordant with polyp testing. The family history met Amsterdam II Criteria for seven patients $(12.3 \%)$, four of whom also had a family history of polyps. A history of LS-associated malignancies not satisfying the Amsterdam II Criteria (Colorectal, Endometrial, Ovarian, and Glioblastoma Multiforme) was identified in 24 patients (42.1\%), 20 patients had a family history of LSassociated malignancies and polyps (35.1\%), and a family history of polyps only was identified five patients $(8.8 \%)$. Six patients $(10.5 \%)$ had no family history of cancer or polyps (Figure 3). The motivation for performing MMR IHC in these six cases is unclear; while two patients had high numbers of polyps ( 25 and 26 per patient); the remaining four had low polyp counts $(2,2,6$ and 7 per patient).

IHC on polyp tissue demonstrated no LOE of MMR proteins in 54 patients (94.7\%). Three polyps demonstrated LOE of MMR proteins; two were TAs and one was a SSA. Subsequent $B R A F^{\mathrm{V} 600 \mathrm{E}}$ somatic testing was positive on the SSA and no germline testing was offered. Germline LS testing was performed in the two patients with MMR-deficient TAs. The first case had loss of MSH6 expression on one of six TAs in a 44 year old man. His sister had been previously diagnosed with endometrioid ovarian cancer at 49 years old, also with LOE of MSH6; she was undergoing germline genetic testing. Her testing identified a MSH6 mutation (NM_000179.2: c.2150_2153delTCAG; p.Val717Alafs*18). He was offered predictive genetic testing and was found to carry the familial MSH6 mutation. In the second case, MMR IHC on one of seven TAs demonstrated LOE of PMS2 in a 74 year old man with a history of colorectal cancer at age 37 years. His family history met the Amsterdam II Criteria; germline testing identified a variant of unknown significance (VUS) in PMS2. The PMS2 VUS (NM_000535.5: c.461G>A; p.Gly154Glu) has been reported once on the LOVD database as of unknown clinical significance, has not been reported in the literature and is 
predicted to be pathogenic by in silico tools. There were no living affected relatives in whom to segregate and tumour tissue from his colorectal cancer was unavailable.

Twenty-three patients/families had available LS-associated tumour tissue for MMR IHC. In 21 of the 23 cases (91.3\%) polyp and tumour MMR IHC were concordant and normal. In the two discordant cases, MMR IHC was normal in the polyps, but demonstrated LOE of at least one MMR protein in tumour tissue. The first case was a 77 year old with 3 TAs; polyp MMR IHC was normal, MMR IHC on a metachronous sebaceous adenoma demonstrated LOE of MSH2/MSH6. Germline testing did not reveal any mutations in MSH2 or MSH6. The second case was a 24 year old woman with a single TA with normal MMR IHC; MMR IHC on her mother's colorectal cancer (diagnosed at 32 years old) and maternal grandmother's colorectal cancer (diagnosed at 75 years old) both demonstrated LOE of MLH1 and PMS2. No germline mutations in the $M L H 1$ gene were detected in her grandmother and subsequent tumour testing demonstrated a $B R A F^{\mathrm{V} 600 \mathrm{E}}$ somatic mutation; her mother's tumour tissue was unavailable for testing and germline genetic testing was not initiated in the patient.

Following polyp MMR IHC, 32 germline genetic tests were offered to 24 patients; 30 tests were carried out with two patients declining testing. LS panel testing (MLH1, MSH2, MSH6, and PMS2) was performed in seven patients, three patients had MUTYH testing, and 13 patients had $M U T Y H$ and $A P C$ testing. Two patients subsequently underwent testing with an extended panel of colorectal cancer and polyposis genes (APC, ATM, AXIN2, BMPR1A, CDH1, CHEK2, GREM1, MLH1, MSH2, MSH6, MUTYH, PMS2, POLD1, POLE, PTEN, SMAD4, STK11, TP53), one subsequently undergoing research-based whole exome sequencing. Five patients underwent genetic testing of non-colorectal cancer or polyposis genes based on family history (1 TP53, 4 BRCA1/BRCA2). Additionally, two patients with 
normal MMR IHC had subsequent MSI testing, demonstrating microsatellite stable adenomas, and two patients had somatic $B R A F^{\mathrm{V} 600 \mathrm{E}}$ testing; both results were concordant with polyp IHC. In this additional testing, only one patient was found to have a pathogenic mutation (bi-allelic MUTYH mutations).

\section{Discussion}

This audit of MMR IHC on colorectal polyps in patients referred to the FCC between 2006 and 2016 identified 57 patients, only three of whom had LOE of MMR proteins; two of these three were subsequently found to carry germline MMR gene variants. In one case genetic testing was already underway in the family and while polyp MMR IHC was concordant with the familial mutation, it did not play a role in the diagnosis of LS. In the second case a germline VUS concordant with polyp IHC was identified, however, the family history was already high risk and this result did not inform care. Thus, while polyp MMR IHC was concordant with germline results, it had a low clinical utility in this cohort. No cases of LS were diagnosed on polyp MMR IHC which would not have been diagnosed by standard testing. Polyp MMR IHC was ineffective in identifying new cases of LS in our cohort; this is consistent with the literature ${ }^{11,12}$.

Two cases were identified with discordant MMR IHC on polyps compared to personal or familial tumour tissue; in both cases the MMR IHC was normal on the polyp tissue but demonstrated LOE of MMR proteins in tumour tissue. In both cases, germline testing did not reveal LS mutations. Germline testing was performed based on tumour MMR IHC, irrespective of the polyp MMR IHC, as is standard practice; therefore the polyp MMR IHC did not influence practice or outcome, and its clinical utility remains low despite its concordance with germline results in these cases. 
The discordance of MMR IHC on polyp compared to tumour tissue, as well as the low detection rate of LOE of MMR IHC on polyps may support an alternative model of cancer development in LS to the adenoma-carcinoma pathway. A non-polyposis pathway for LS colorectal cancers is supported by the recent finding of MMR-deficient crypts, with minimal architectural or cytological differences from MMR-proficient crypts, in LS patients ${ }^{15,16}$. If MMR-deficient crypts are the pre-malignant lesion this could explain the colorectal cancer risk in the generally non-polyposis LS phenotype and challenge the traditional adenomacarcinoma model where MMR inactivation occurs after adenoma formation, suggesting that LOE of MMR proteins may occur independently of polyp formation ${ }^{17}$. If LS-associated cancers occur independent of the adenoma-carcinoma pathway, the utility of MMR IHC on polyps is likely to be low. This alternate pathway may also explain the occurrence of interval colorectal cancers in LS patients compliant with colonoscopy screening ${ }^{14}$.

Twenty-four patients (42.1\%) underwent 30 germline genetic tests in our study, and 21 of these 24 had normal polyp MMR IHC, inferring that the polyp MMR IHC did not provide sufficient clinical reassurance to negate the need for germline testing and questioning the clinical utility and value of MMR IHC. While MMR IHC is a relatively inexpensive test, there are significant hidden costs in retrieving and slicing of archival blocks. Even when these costs are low, if a normal result does not adequately inform decision making and negate further testing, then MMR IHC is a poor value test. As the cost of genetic testing rapidly declines and the availability of multi-gene panel testing increases, the cost of MMR IHC and germline testing are likely to converge. Additionally, in the scenario of archival tissue from an external pathology provider being required the costs of MMR IHC and germline genetic testing are already comparable. 
Performing MMR IHC is useful in directing germline genetic testing, but with the decrease in single gene testing for LS, MMR IHC is less clinically relevant; although, the MMR IHC directed single gene approach may reduce the risk of incidental findings, which can complicate multi-gene approaches. Additionally, the case can still be made for MMR IHC testing of LS-associated malignant tissue to inform variant classification. Given the more definitive nature of germline genetic testing over MMR IHC, particularly when performed on non-malignant polyp tissue with unknown sensitivity and specificity, we found minimal clinical utility in performing polyp MMR IHC.

Given the low clinical utility and value of polyp MMR IHC, which has been previously demonstrated in the literature $5,9,11,12$ and confirmed in this study, the clinical practice of ordering such testing should be examined. In our cohort, MMR IHC testing was requested by multiple FCC-associated clinicians including geneticists, gastroenterologists and medical oncologists. The clinical rational for testing was poorly documented in the majority of cases; however, review of the identified cases indicates it may have been requested based on a personal or family history of LS-associated cancers in the absence of available tumour tissue. While the desire to offer some form of testing in these cases is appreciable, the poor value and low utility of polyp MMR IHC confirms its use is not justified. However, the generalisation of these results is limited by this study's relatively small size and single centre retrospective nature.

\section{Conclusion}

Screening of polyps with MMR IHC was ineffective in identifying new cases of LS in this study, which is consistent with the published literature ${ }^{11,12}$, and its use in mitigating the need for further genetic testing was inadequate thus making its clinical utility low. Given the cost 
associated with MMR IHC in comparison with the rapidly decreasing costs of germline genetic testing, we also found there was minimal clinical value in polyp MMR IHC. Given the low value and utility, both in this and other published studies, we recommend against the use of MMR IHC in the Familial Cancer Clinic and more generally, as directed by the Royal Australasian College of Physician's Evolve Program ${ }^{13}$.

\section{Acknowledgements}

No funding was received to support this study. The authors declare that they have no competing interests and acknowledge no other contributors to this work. 


\section{References}

1 Rustgi AK. The genetics of hereditary colon cancer. Genes Dev 2007; 21(20): 2525-2538.

2 Boland CR, Goel A. Microsatellite instability in colorectal cancer. Gastroenterology 2010; 138(6): 2073-2087.

3 Jass JR, Stewart SM, Stewart J, Lane MR. Hereditary non-polyposis colorectal cancer-morphologies, genes and mutations. Mutat Res 1994; 310(1): 125-133.

4 Walsh MD, Buchanan DD, Pearson SA, Clendenning M, Jenkins MA, Win AK et al. Immunohistochemical testing of conventional adenomas for loss of expression of mismatch repair proteins in Lynch syndrome mutation carriers: a case series from the Australasian site of the colon cancer family registry. Mod Pathol 2012; 25(5): 722-730.

5 Mendelsohn RB, Herzog K, Shia J, Rahaman N, Stadler ZK, Shike M. Molecular Screening for Lynch Syndrome in Young Patients With Colorectal Adenomas. Clin Colorectal Cancer $2017 ; 16(3): 173-177$.

6 Pino MS, Mino-Kenudson M, Wildemore BM, Ganguly A, Batten J, Sperduti I et al. Deficient DNA mismatch repair is common in Lynch syndrome-associated colorectal adenomas. J Mol Diagn 2009; 11(3): 238-247.

7 Yurgelun MB, Goel A, Hornick JL, Sen A, Turgeon DK, Ruffin MT 4th et al. Microsatellite instability and DNA mismatch repair protein deficiency in Lynch syndrome colorectal polyps. Cancer Prev Res (Phila) 2012; 5(4): 574-582.

8 Iino H, Simms L, Young J, Arnold J, Winship IM, Webb SI et al. DNA microsatellite instability and mismatch repair protein loss in adenomas presenting in hereditary nonpolyposis colorectal cancer. Gut 2000; 47(1): 37-42.

9 Loukola A, Salovaara R, Kristo P, Moisio AL, Kääriäinen H, Ahtola H et al. Microsatellite instability in adenomas as a marker for hereditary nonpolyposis colorectal cancer. Am J Pathol 1999;155(6):1849-1853. 
10 Ferreira S, Claro I, Lage P, Filipe B, Fonseca R, Sousa R et al. Colorectal adenomas in young patients: microsatellite instability is not a useful marker to detect new cases of Lynch syndrome. Dis Colon Rectum 2008; 51(6): 909-915.

11 Velayos FS, Allen BA, Conrad PG, Gum J Jr, Kakar S, Chung DC et al. Low rate of microsatellite instability in young patients with adenomas: reassessing the Bethesda guidelines. Am J Gastroenterol 2005; 100(5): 1143-1149.

12 Koh DC, Luchtefeld MA, Kim DG, Attal H, Monroe T, Ingersoll K. Microsatellite instability and MLH1 hypermethylation - incidence and significance in colorectal polyps in young patients. Colorectal Dis 2007; 9(6): 521-526.

13 Soon J, Buchbinder R, Close J, Hill C $C^{4}$, Allan $S^{5}$, Turnour C. Identifying low-value care: the Royal Australasian College of Physicians' EVOLVE initiative. Med J Aust 2016; 204(5): 180-181.

14 Levinson W, Kallewaard M, Bhatia RS, Wolfson D, Shortt S, Kerr EA et al. 'Choosing Wisely': a growing international campaign. BMJ Qual Saf 2015; 24(2): 167-174.

15 Ahadova A, von Knebel Doeberitz M, Blaker H, Kloor M. CTNNB1-mutant colorectal carcinomas with immediate invasive growth: a model of interval cancers in Lynch syndrome. Fam Cancer 2016; 15(4): 579-586.

16 Kloor M, Huth C, Voigt AY, Benner A, Schirmacher P, von Knebel Doeberitz M et al. Prevalence of mismatch repair-deficient crypt foci in Lynch syndrome: a pathological study. Lancet Oncol 2012; 13(6): 598-606.

17 Shia J, Stadler ZK, Weiser MR, Vakiani E, Mendelsohn R, Markowitz AJ et al. Mismatch repair deficient-crypts in non-neoplastic colonic mucosa in Lynch syndrome: insights from an illustrative case. Fam Cancer 2015; 14(1): 61-68. 
Figure 1. Flow chart of inclusion/exclusion criteria applied to patient cohort (IHC immunohistochemistry, MMR - mismatch repair, LOE - loss of expression).

Figure 2. Subtype of polyp on which mismatch repair immunohistochemistry was performed (IHC - immunohistochemistry).

Figure 3. Family history of patients undergoing polyp mismatch repair immunohistochemistry. 


\begin{abstract}
Background: Immunohistochemistry for loss of expression of one or more of the mismatch repair proteins is performed on colorectal cancer tissue as a screening test for Lynch syndrome; however, its role in premalignant polyps remains controversial.

Aim: To determine the effectiveness of mismatch repair immunohistochemistry performed on premalignant colorectal polyps in identifying Lynch syndrome, focusing on clinical utility and value.
\end{abstract}

Methods: A retrospective audit was conducted of mismatch repair immunohistochemistry performed on non-malignant polyps in patients who attended the Family Cancer Clinic at the Royal Melbourne Hospital. Two hundred and six patient records over a 10 year period (20062016) were reviewed. Personal and family history data was collected, including genetic testing results.

Results: Of the 57 patients who underwent polyp testing, the family histories comprised Amsterdam II Criteria (12.3\%), Lynch syndrome-associated malignancies (42.1\%), Lynch syndrome-associated malignancies and polyps (35.1\%), and polyps only $(8.8 \%) ; 10.5 \%$ of patients had no significant family history. Normal expression of the mismatch repair proteins was observed in $94.7 \%$ of patients; loss of expression was observed in 3 individuals with concordant germline variants in 2 patients (1 PMS2 variant of unknown significance and 1 MSH6 mutation). Additional genetic testing in 21 patients with normal immunohistochemistry did not identify any additional Lynch syndrome cases.

Conclusion: The clinical utility of mismatch repair immunohistochemistry on polyp tissue was low. No additional cases of Lynch syndrome were identified, and a large proportion of patients proceeded to germline testing despite normal polyp immunohistochemistry. We suggest there is no value in this approach. 


\section{University Library}

\section{- M M N E R VA A gateway to Melbourne's research publications}

Minerva Access is the Institutional Repository of The University of Melbourne

Author/s:

Dow, E;Buchanan, DD;Winship, IM

Title:

Utility of immunohistochemistry for mismatch repair proteins on colorectal polyps in the familial cancer clinic

Date:

2018-11-01

Citation:

Dow, E., Buchanan, D. D. \& Winship, I. M. (2018). Utility of immunohistochemistry for mismatch repair proteins on colorectal polyps in the familial cancer clinic. INTERNAL MEDICINE JOURNAL, 48 (11), pp.1325-1330. https://doi.org/10.1111/imj.13953.

Persistent Link:

http://hdl.handle.net/11343/283959 